\title{
Female urinary incontinence and wellbeing: results from a multi-national survey
}

\author{
Andrew P. Smith
}

\begin{abstract}
Background: Previous research has shown that the severity of symptoms of urinary incontinence impacts on quality of life and wellbeing.

The aim of this article is to investigate the relationship between female urinary incontinence and mental wellbeing. This involved analyses comparing those with $\mathrm{UI}$ and those without to determine whether any differences in wellbeing were modified by demographic factors, specific wellbeing domain, or exercise and frequency of sex. Following this, further analyses compared sub-groups of those with UI (based on the impact of the UI) to determine which characteristics were important in influencing wellbeing.

Methods: An internet survey of women with UI, aged between 45 and 60 years, has been previously reported and this article reports secondary analyses of that data.

A sample from 4 countries: the UK, France, Germany and the USA.

Two thousand four hundred three women completed the survey, 1203 with UI and 1200 who did not report UI. The main outcome measures were the scores from the Warwick-Edinburgh Mental Wellbeing Scale (WEMWBS).

Results: The results showed that lower wellbeing is observed in UI. This effect is observed in all aspects of wellbeing and most sub-groups of UI sufferers. Lifestyle influences wellbeing and those with UI who exercise less frequently or have sex infrequently are especially likely to report lower wellbeing. Wellbeing decreases as a function of the indirect measures of severity of $\mathrm{UI}$ and reductions in HRQol. Again, these changes reflect all aspects of wellbeing measured by WEMWBS.
\end{abstract}

Conclusions: The results show that women with UI, aged 45-60 years, report lower wellbeing. This effect was not modified by demographic factors and was apparent in most of the domains measured by the WEMWBS. The reduced wellbeing was related to the impact of the UI on behaviour, embarrassment associated with it, and frequency of leakage.

Keywords: Urinary incontinence, Wellbeing, Female, Quality of life, Exercise, Sex

\section{Background}

The prevalence of urinary incontinence (UI) in adult women has been estimated to be about $18 \%$ [1] and in the elderly this figure can be as high as $55 \%$ [2]. UI can negatively affect many aspects of life, and has been shown to affect overall health-related quality of life (HRQoL) [3-12]. Indeed, a search of PubMed reveals 28 articles that have examined UI and HRQol. A recent survey [13] aimed to develop a clear understanding of the impact of urinary incontinence on the quality of life

Correspondence: smithap@cardiff.ac.uk

Centre for Occupational and Health Psychology, School of Psychology,

Cardiff University, 63 Park Place, Cardiff CF10 3AS, UK
(Qol) of women of working age. The results showed that the severity of symptoms of UI impacted on quality of life. The results also showed that wellbeing decreased as the symptoms of UI became more severe. The relationship between HRQol and wellbeing was also examined and it was found that UI reduced HRQol which in turn led to reduced wellbeing.

\section{Wellbeing}

Historically, research on wellbeing has mainly been conducted within two traditions: hedonic wellbeing and eudaimonic wellbeing. The hedonic approach to wellbeing measures subjective wellbeing which is comprised 
of life satisfaction, positive affect, and an absence of negative affect. Specifically, focusing on happiness from the perspective of pleasure versus pain including both cognitive evaluation (life satisfaction) and affect (with positive and negative affect comprising separate dimensions). In contrast, the eudaimonic approach to wellbeing is not simply interested in subjective happiness, but in the realisation of human potential. Within this view, wellbeing is linked to a person living in a way which is congruent with their deeply held values: a meaningful life characterised by personal growth, as opposed to a pleasurable life characterised by hedonic enjoyment. There is now international interest in wellbeing and its role in all aspects of life. This interest can be seen at a population level and in specific contexts such as work. In addition, wellbeing is now an outcome of interest in many health-related areas. Wellbeing scales such as the Warwick-Edinburgh Wellbeing Scale (WEMWBS;) [14] measure both hedonic and eudaimonic wellbeing and are correlated with general health, positive and negative affect, life satisfaction and mental ill-health. UI has been shown to negatively impact on these outcomes and it is important to investigate whether wellbeing scores are related to UI as at the moment there is little information on this topic.

\section{Wellbeing and UI}

There have been a number of studies which have investigated associations between incontinence and mental health [15-17]. These generally confirm that UI is associated with an increased risk of depression [15], and show that treatment of the depression associated with UI may be an effective way of reducing the burden of UI [16]. Indeed, interventions aimed at increasing resilience may lessen the impact of depression on those with UI [17].

Two Australian studies have investigated associations between UI and wellbeing $[18,19]$. The first study [18] showed that UI was associated with reduced wellbeing but that the relationship between different types of UI and wellbeing was different. The second study [19] investigated associations between UI and wellbeing in young nulligravid women. The results confirmed that women with UI reported lower wellbeing that women without UI. It is now important to replicate and extend these findings using multi-national samples of women of working age and recently developed measures of wellbeing.

\section{Aims}

The primary aim was to conduct secondary analyses of our previous study [13] which focused on UI in women of working age to provide a detailed profile of female UI and wellbeing. This involved comparison of the wellbeing of those with UI with those with no symptoms.
Examination of the individual items of the WEMWBS was carried out to determine whether effects are global or restricted/greatest in specific domains. Further analyses examined whether the associations between UI and wellbeing (and effects on wellbeing of those without UI) varied as a function of demographics and lifestyle (frequency of exercise and sex). Our previous paper from this survey [13] focused on associations between UI severity, HRQoL and WEMBS. UI symptoms were associated with lower HRQoL, which then impacts negatively on wellbeing. In the analyses reported here sub-sets of the UI group (based on indicators of severity - e.g., frequency of wearing pads; interference with activities) were also compared. The general hypothesis being tested was that even relatively mild UI would be associated with reduced wellbeing (compared to those without UI) and that such effects would be global rather than restricted to specific domains or sub-groups. Additionally, the analyses examined whether there was variation within the UI group as a function of the impact of UI on different activities and outcomes. These secondary analyses extend the initial aims of the protocol and address issues that are of practical relevance to women of working age with UI.

\section{Method}

The survey was conducted by Ipsos Market Research and adhered to the Market Research Society code of conduct and was carried out with the informed consent of the participants. The Ipsos samples are representative of the country in terms of demographics and are selected using the recruitment criteria of the specific survey (see below).

\section{Study population}

An internet survey (with the questions in the appropriate language) was conducted in 4 countries (France, Germany, UK and USA) during September 2013. A sample of women aged 45-60 years was recruited by Ipsos Marketing. Their online panels currently consist of approximately 920,000 members in the USA, 535,000 in France, 300,000 in the UK, and 169,000 in Germany. The survey was answered by both women with and without UI and the sampling procedure meant that the UI and non-UI groups did not differ in terms of basic demographics. Informed consent was represented by participation in the survey after the provision of the information about the study. Participants were aware of this procedure when they joined the online panels. The first 300 respondents with UI in each of the four countries were included in the study population, as were the first 300 non-UI respondents. This procedure meant that it was impossible to determine how many would have completed the survey if there was no sample cut-off point nor how many would have refused to participate. 
Sample size was based on the need to detect small effect sizes (Cohen's $\mathrm{d}=0.2$; with power set at 0.9 and $p=0.05$ a total sample size of 1054 would be needed) in the wellbeing outcomes. Participants were paid for participating in the study and after this the database was anonymised.

\section{Survey questionnaire}

UI categorisation was defined by the answer to the ICIQ-UI Short Form question "how often do you leak urine?; those answering "Never" were classed as without UI while all other answers were classed as having UI. UI severity was based on the response to "How much urine do you usually leak?" [20] with a small amount being classified as "light UI", a moderate amount as "medium UI" and a large amount as "severe UI". Those reporting UI completed questions measuring UI symptoms (ICIQ-UI short form) [21] and UI quality of life (ICIQ-LUTSqol) [20]. Both UI and non-UI participants completed the WEMWBS [14] which has 14 items and a Likert scale of 1-5 giving a maximum score of 70 .

Demographic factors (e.g., age; marital status) were recorded as were frequency of exercise and sex. Other questions examined the impact of UI on aspects of life. Outcomes of UI were also recorded (degree of embarrassment; frequency of wearing pads). The complete set of questions are given in supplementary information with our previous publication from the study [13].

\section{Statistical analysis}

The first set of analyses compared those reporting UI with those who did not. Analyses were carried out on the individual items of the wellbeing scale to determine whether any specific domains were more impaired than others. Sub-groups based on demographic factors were also compared. The initial analyses involved a MANOVA to determine whether effects were apparent across all items of the wellbeing scale. Most studies use the WEMWBS total score but analysis of the individual items provided an opportunity to examine the generality of effects. Subsequent analyses examined whether effects were apparent in subgroups of patients (based on demographics, frequency of exercise and sex) and used univariate ANOVAs followed by multiple regression. Factor analyses were carried out to identify categories of outcomes associated with UI. ANOVAs were then carried out to examine changes in wellbeing as a function of the severity of these different types of problem. A similar approach was used to examine the effects of factors leading to leakage on wellbeing. The analyses were carried out using the IBM SPSS Statistics 20 package.

\section{Results}

There was no missing data for the variables considered in the initial analyses comparing those reporting UI with the non-UI group. Those with UI reported significantly lower wellbeing (UI mean: 48.2 s.d. 10.2; Non-UI mean: 50.2 s.d. $8.9 ; \mathrm{t}=5.3$ d.f. $2401 p<0.001$ ) and a multivariate analysis of variance showed this was significant for all items in the scale (Wilks Lambda $=0.97 p<0.001$; see Table 1 for means and s.d.s.

All of the individual effects remained significant when a Holm-Bonferroni correction for multiple comparisons was applied. Subsequent analyses examined whether the effect varied as a function of country, age and marital status. The results (see Table 2) showed that the effect of UI was observed in the majority of sub-groups groups (the exceptions being the French and those who were widowed). There was no missing data in these analyses.

A multi-variate analysis was then carried out in which UI v non-UI, country, age, marital status, frequency of exercise and frequency of sex were entered into a

Table 1 Scores for individual wellbeing questions (higher scores $=$ greater wellbeing)

\begin{tabular}{|c|c|c|c|c|}
\hline Question & Group & Mean & SD & Significance \\
\hline \multirow[t]{2}{*}{ Feeling cheerful } & $\mathrm{UI}$ & 3.39 & 0.91 & $F=17.0 p<0.001$ \\
\hline & Non-UI & 3.53 & 0.84 & \\
\hline \multirow[t]{2}{*}{ Thinking clearly } & UI & 3.71 & 0.95 & $F=12.2 p<0.001$ \\
\hline & Non-UI & 3.84 & 0.89 & \\
\hline \multirow[t]{2}{*}{ Close to other people } & UI & 3.45 & 0.99 & $F=17.0 p<0.001$ \\
\hline & Non-UI & 3.61 & 0.87 & \\
\hline \multirow[t]{2}{*}{ Feeling confident } & UI & 3.35 & 0.97 & $F=27.0 p<0.001$ \\
\hline & Non-UI & 3.54 & 0.90 & \\
\hline \multirow[t]{2}{*}{ Dealing with problems well } & UI & 3.58 & 0.88 & $F=12.0 p<0.001$ \\
\hline & Non-UI & 3.70 & 0.81 & \\
\hline \multirow[t]{2}{*}{ Energy to spare } & $\mathrm{UI}$ & 2.79 & 1.05 & $F=44.8 p<0.001$ \\
\hline & Non-UI & 3.06 & 0.94 & \\
\hline \multirow[t]{2}{*}{ Feeling good about myself } & $\mathrm{UI}$ & 3.35 & 0.96 & $F=19.0 p<0.001$ \\
\hline & Non-UI & 3.51 & 0.87 & \\
\hline \multirow[t]{2}{*}{ Interested in new things } & UI & 3.46 & 1.00 & $F=13.4 p<0.001$ \\
\hline & Non-UI & 3.60 & 0.88 & \\
\hline \multirow[t]{2}{*}{ Interested in other people } & UI & 3.56 & 0.98 & $F=9.9 p<0.001$ \\
\hline & Non-UI & 3.68 & 0.87 & \\
\hline \multirow[t]{2}{*}{ Feeling loved } & $\mathrm{UI}$ & 3.56 & 1.09 & $F=7.8 p<0.005$ \\
\hline & Non-UI & 3.68 & 1.02 & \\
\hline \multirow[t]{2}{*}{ Feeling optimistic about future } & $\mathrm{UI}$ & 3.25 & 1.06 & $F=7.3 p<0.01$ \\
\hline & Non-UI & 3.36 & 0.96 & \\
\hline \multirow[t]{2}{*}{ Able to make up own mind } & $\mathrm{UI}$ & 4.03 & 0.88 & $F=12.1 p<0.005$ \\
\hline & Non-UI & 4.15 & 0.79 & \\
\hline \multirow[t]{2}{*}{ Feeling relaxed } & UI & 3.21 & 0.95 & $F=6.7 p<0.01$ \\
\hline & Non-UI & 3.31 & 0.89 & \\
\hline \multirow[t]{2}{*}{ Feeling useful } & UI & 3.46 & 0.98 & $F=25.0 p<0.001$ \\
\hline & Non-Ui & 3.66 & 0.89 & \\
\hline
\end{tabular}


Table 2 Total wellbeing scores in the $\mathrm{UI}$ and non-UI groups as a function of country, age and marital status (high scores = greater wellbeing)

\begin{tabular}{llll}
\hline Group & Mean & SD & N \\
\hline USA UI & 48.2 & 10.9 & 300 \\
USA non-UI & 51.9 & 9.9 & 300 \\
UK UI & 46.1 & 10.2 & 300 \\
UK non-UI & 48.8 & 9.2 & 301 \\
Germany UI & 49.7 & 10.2 & 301 \\
Germany non-UI & 51.6 & 8.6 & 300 \\
France UI & 48.7 & 9.4 & 301 \\
France non-UI & 48.7 & 7.3 & 300
\end{tabular}

Country $\times$ UI group interaction: $F(3,2395)=3.8 p<0.05$

Age

$\begin{array}{llll}45-48 \text { years UI } & 47.6 & 9.9 & 262 \\ 45-48 \text { non-UI } & 49.4 & 9.2 & 292 \\ 49-53 \text { years UI } & 47.4 & 10.3 & 367 \\ 49-53 \text { non-UI } & 49.8 & 8.5 & 342 \\ 54-57 \text { years UI } & 48.8 & 10.8 & 247 \\ 54-57 \text { non-UI } & 51.2 & 8.8 & 233 \\ 58-60 \text { years UI } & 49.0 & 10.1 & 327 \\ 58-60 \text { years non- UI } & 50.8 & 9.1 & 333\end{array}$

Age $\times$ UI group interaction: $F<1$

Marital Status

$\begin{array}{llll}\text { Single UI } & 47.0 & 9.5 & 164 \\ \text { Single non-UI } & 48.3 & 8.5 & 202 \\ \text { Married/living together UI } & 49.0 & 9.9 & 743 \\ \text { Married non-UI } & 51.0 & 8.9 & 695 \\ \text { Widowed UI } & 47.7 & 8.9 & 51 \\ \text { Widowed non-UI } & 46.9 & 10.4 & 49 \\ \text { Divorced/separated UI } & 46.6 & 11.8 & 245 \\ \text { Divorced non-UI } & 50.3 & 8.7 & 254 \\ \text { Marital status x UI group interaction: F 93,2395) }=2.1 p>0.05)\end{array}$

regression with wellbeing as the outcome. All of the effects, except marital status, were significant (see Table 3) confirming the results of the individual analyses.

The next set of analyses examined effects of frequency of exercise (no missing data) and sex (507 participants preferred not to answer this question, with there being

Table 3 Multi-variate regression with total WEMWBS score as the dependent variable

\begin{tabular}{llllll}
\hline Variable & B & Std error & Standardised B & t & \multicolumn{1}{l}{ Sig } \\
\hline Age & 1.557 & 0.359 & .086 & 4.334 & $<0.001$ \\
Exercise & -1.596 & .163 & -.194 & -9.784 & $<0.001$ \\
Sex & -.684 & .122 & -.111 & -5.588 & $<0.001$ \\
UI v non-UI & 1.886 & .356 & .105 & 5.302 & $<0.001$ \\
\hline
\end{tabular}

significantly more in this category in the non-UI group) on wellbeing in both groups. Reduced wellbeing in the UI group was greatest in those who did not frequently exercise. Frequency of having sex was associated with greater wellbeing and this was true for both UI and nonUI groups (see Table 4).

The next set of analyses considered the impact of problems associated with UI. Factor analysis showed three categories of problem: Relationships (sex; social life); Work/household/physical activity; and Fatigue/mental health. On the basis of these factor scores the UI sample was split into tertiles in order to provide an initial indication of dose-response. As problems increased, wellbeing decreased (mainly in the last tertile - see Table 5), although the effects were bigger for relationships and fatigue/mental health than the work/household/physical category.

Wellbeing also decreased as a function of the degree of embarrassment associated with UI $(F(2,1200)=68.7$ $p<0.001$ : Never embarrassed: mean $=51.8$ s.d. $=9.3$; Sometimes embarrassed: mean $=47.6$ s.d. $=9.3$; Often $/$ All the time: mean $=41.9$ s.d. $=11.6$ ). A fourth set of analyses examined when they leaked and the use of pads. Factor analyses $(N=1177)$ showed three categories of leakage: No obvious reason; Cough/sneeze/exercise; and before getting to the toilet. On the basis of these factor scores the UI sample were split into tertiles. An increase in all these types of leakage was associated with reduced wellbeing (see Table 6) as was frequency of wearing pads $(F(3,1199)=5.1 p<0.005$ : Never: mean $=50.5$ s.d. $=9.2$; Sometimes: mean $=48.6$ s.d. $=10.2$; Often $/$ All the time: mean $=47.1$ s.d. $=10.5)$. Frequency of using pads was associated with more severe UI which in turn was

Table 4 Frequency of exercise and sex and wellbeing (high scores = greater wellbeing; More exercise = every day or once or twice a week; Less exercise = once or twice a month or never. More frequent sex = once or twice a week or more; Less frequent sex = once or twice a month or I can't remember when)

\begin{tabular}{llll}
\hline Group & Mean & SD & N \\
\hline UI more exercise & 49.7 & 10.0 & 806 \\
Ul less exercise & 45.1 & 10.2 & 397 \\
Non-Ul more exercise & 51.1 & 8.7 & 835 \\
Non-Ul less exercise & 48.2 & 9.2 & 365
\end{tabular}

Main effect of exercise: $F(1,2399)=82.8 p<0.001$

Exercise $x$ UI interaction: $F(1,2399)=4.2 p<0.05$

\begin{tabular}{lcll} 
UI more sex & 51.4 & 9.6 & 357 \\
Ul less sex & 45.9 & 10.4 & 631 \\
Non-Ul more sex & 53.1 & 8.0 & 369 \\
Non-UI less sex & 48.0 & 9.4 & 539 \\
Main effect of sex: $F(1,1892)=145.3 p<0.001)$ & & \\
Sex $x$ UI interaction: $F<1$ & & \\
\hline
\end{tabular}


Table 5 Wellbeing scores (mean, s.d,) and impact of UI (shown as tertiles, tertile $1=$ lowest impact)

\begin{tabular}{lllll}
\hline Factor & T1 & T2 & $T 3$ & \\
\hline Relationships & 49.79 .2 & 51.39 .5 & 45.510 .9 & $F 2,978=30.2 p<0.001$ \\
Work/household/physical activities & 49.910 .1 & 48.99 .5 & 47.710 .9 & $F 2,978=4.0 p<0.05$ \\
Mental health & 52.49 .1 & 49.79 .7 & 44.410 .1 & $F 2,978=58.0 p<0.001$ \\
\hline
\end{tabular}

associated with lower wellbeing (Light UI: $N=$ mean $=$ 49.4 s.d. $=9.5 ;$ Medium UI: mean $=44.6$ s.d. $=11.4$; Severe UI: mean $=42.2$ s.d. $=12.7$ ).

\section{Discussion}

These results showed that reduced wellbeing is observed in UI. This effect is observed in all aspects of wellbeing and most sub-groups of UI sufferers. Factors which reflect greater severity of UI lead to a greater reduction in wellbeing. Lifestyle influences wellbeing and those with UI who exercise less frequently or have sex infrequently are especially likely to report reduced wellbeing. Wellbeing decreases as a function of the severity of UI and reductions in HRQol. Again, these changes reflect all aspects of wellbeing measured by WEMWBS. Previous analyses [13] showed that when both quality of life (ICIQ-LUTSqol) and symptom severity (ICIQ-UI Short Form) were included in the same regression analysis, the only significant effect on mental well-being was ICIQLUTSqol. This shows that UI symptoms do not directly affect mental well-being, but that symptoms influence quality of life, which in turn influences well-being.

This is the first multi-national study which has examined the impact of UI on wellbeing. The sample consisted of women aged 45-60 in four developed countries and this population enables assessment of wellbeing in a group with a busy active life which is likely to be more demanding than that of elderly populations who are often studied in UI research. The present sample consisted of women who largely reported light UI and yet the results show a clear reduction in wellbeing in this group. Those with severe incontinence find it extremely difficult to engage in sex and physical exercise but by sampling those with light UI the present study was able to look at how those activities related to wellbeing in both UI and non-UI groups. Improving wellbeing in those with mild UI is clearly very important and shows that clinical management must address this as well as the issue of leakage. UI is often under reported and community education is desirable as those with mild UI may not see a clinician. It is also important to investigate the extent to which individuals adopt compensatory behaviours which enable them to function and minimize the problems related to UI.

The study has a number of limitations and further research is required to extend the present findings and reduce any potential biases present in this study. The online format of the survey may have excluded certain groups and future research should consider a casecontrol approach to the comparison of UI and non-UI groups. Type of incontinence (stress, urgency and mixed) is also a factor which can now be assessed using wellbeing outcomes. More specific information about exercise and sexual behaviour is also required, as is information on BMI which may be important in UI and wellbeing. Other psychosocial factors known to influence wellbeing (e.g., personality; stress; social support and coping) should also be measured in order to determine whether the effects of UI and these psychosocial factors have independent, additive or interactive effects on wellbeing. The practical significance of the small difference in wellbeing also needs to be assessed and bench marked against effect sizes seen in other chronic conditions. The WEMWBS was not really designed to determine clinical significance and other measuring instruments should be also used to determine whether any differences are clinically significant.

\section{Conclusion}

In conclusion, the results from the present survey demonstrate that even light UI is associated with lower wellbeing. This appears to be a general problem associated with UI in that it is present in most demographic groups and is observed in all the domains measured by the WEMWBS. Within the UI group the lower wellbeing was related to HRQol and to perceptions of interference with specific activities or the induction of mental health issues. Therapies aimed at increasing wellbeing (e.g., mindfulness; Cognitive Behaviour Therapy) may play a role in the management of UI.

Table 6 Reason for leakage (shown as tertiles; $\mathrm{T} 1$ = not present) and wellbeing (mean, s.d., N)

\begin{tabular}{lllll}
\hline Factor & T1 & T2 & T3 & \\
\hline No obvious reason & 50.09 .0 & 49.09 .2 & 44.811 .5 & $F 2,1174=29.9 p<0.001$ \\
Cough/sneeze/exercise & 48.410 .7 & 48.610 .3 & 47.09 .6 & $F 2,1174=2.9 p<0.05$ \\
Before getting to toilet & 48.910 .5 & 48.89 .4 & 46.210 .7 & $F 2,1174=8.7 p<0.001$ \\
\hline
\end{tabular}




\section{Acknowledgements}

N/A.

\section{Author's contribution}

Professor Smith designed and analysed the wellbeing part of the study. He also wrote the article.

\section{Availability of data and materials}

Supporting material can be found at: http://onlinelibrary.wiley.com/doi/ 10.1111/bju.12852/full

\section{Funding}

This study was funded by Procter and Gamble Ltd.

\section{Competing interests}

Professor Smith has received an honorarium from Procter and Gamble Ltd.

\section{Consent to publish}

This was obtained at recruitment.

\section{Ethical approval and consent to participate}

The research described in this article was originally conceptualised as market research and adhered to the Market Research Society Code of conduct. Given this, ethical approval was not sought prior to data collection. Informed consent was represented by participation in the survey after the provision of the information about the study. Participants were aware of this procedure when they joined the online panels.

Received: 13 January 2016 Accepted: 16 May 2016

Published online: 23 May 2016

\section{References}

1. Irwin DE, Milsom I, Hunskaar S, Reilly K, Kopp Z, Herschorn S, Coyne K, Kelleher C, Hampel C, Artibani W, et al. Population-based survey of urinary incontinence, overactive bladder, and other lower urinary tract symptoms in five countries: results of the EPIC study. Euro Urol. 2006:50(6):1306-14. discussion 1314-1305.

2. Thom D. Variation in estimates of urinary incontinence prevalence in the community: effects of differences in definition, population characteristics, and study type. J Am Geriatr Soc. 1998;46(4):473-80.

3. Franzen K, Johansson JE, Andersson G, Pettersson N, Nilsson K. Urinary incontinence in women is not exclusively a medical problem: a populationbased study on urinary incontinence and general living conditions. Scand J Urol Nephrol. 2009;43(3):226-32.

4. Fultz NH, Fisher GG, Jenkins KR. Does urinary incontinence affect middleaged and older women's time use and activity patterns? Obstet Gynecol. 2004;104(6):1327-34

5. Coyne KS, Sexton CC, Irwin DE, Kopp ZS, Kelleher CJ, Milsom I. The impact of overactive bladder, incontinence and other lower urinary tract symptoms on quality of life, work productivity, sexuality and emotional well-being in men and women: results from the EPIC study. BJU Int. 2008;101(11):1388-95.

6. Papanicolaou S, Hunskaar S, Lose G, Sykes D. Assessment of bothersomeness and impact on quality of life of urinary incontinence in women in France, Germany, Spain and the UK. BJU Int. 2005:96(6):831-8

7. Broome BA. The impact of urinary incontinence on self-efficacy and quality of life. Health Qual Life Outcomes. 2003;1:35

8. Sinclair AJ, Ramsay IN. The psychosocial impact of urinary incontinence in women. Obstet Gynaecol. 2011:13(3):143-8.

9. Tang DH, Colayco DC, Khalaf KM, Piercy J, Patel V, Globe D, Ginsberg D. Impact of urinary incontinence on healthcare resource utilization, health-related quality of life and productivity in patients with overactive bladder. BJU Int. 2014;113:484-91.

10. Coyne KS, Wein A, Nicholson S, Kvasz M, Chen Cl, Milsom I. Comorbidities and personal burden of urgency urinary incontinence: a systematic review. The Int J Clin Pract. 2013:67:1015-33.

11. Osman NI, Chapple CR. The burden of urgency urinary incontinence on health and wellbeing. The Int J Clin Pract. 2013;67:1072-4.

12. Coyne KS, Wein A, Nicholson S, Kvasz M, Chen Cl, Milsom I. Economic burden of urgency urinary incontinence in the United States: a systematic review. J Manag Care Pharm. 2014;20:130-40.
13. Abrams $P$, Smith AP, Cotterill N. The impact of urinary incontinence on quality of life in a real-world population of women aged 45-60 years: results from a survey in France, Germany, the UK and the USA. Br J Urol Int. 2014. doi:10.1111/bju.12852.

14. Tennant R, Hiller L, Fishwick R, Platt S, Joseph S, Weich S, Parkinson J, Secker J, Stewart-Brown S. The Warwick-Edinburgh Mental Well-being Scale (WEMWBS): development and UK validation. Health Qual Life Outcomes. 2007;5:63.

15. Avery J, Braunack-Mayer A, Stocks N, Taylor A, Duggan P. Psychological perspectives in urinary incontinence: a metasynthesis. OA Women's Health. 2013;1:9.

16. Avery J, Stocks N, Duggan P, Braunack-Mayer A, Taylor A, Goldney R, MacLennan A. Identifying the quality of life effects of urinary incontinence with depression in an Australian population. BMC Urol. 2013;13. doi:10.1186/1471-2490-13-11.

17. Avery JC, Braunack-Mayer AJ, Duggan PM, Taylor AW, Stocks NP. 'It's our lot': how resilience influences the experience of depression in women with urinary incontinence. Health Sociol Rev. 2015;24:94-108.

18. Botlero R, Bell RJ, Urquhart DM, Davis SR. Urinary incontinence is associated with lower psychological general well-being in community-dwelling women. Menopause. 2010;17:332-7.

19. O'Halloran T, Bell RJ, Robinson PJ, Davis SR. Urinary incontinence in young nulligravid women. Ann Intern Med. 2012;157:87-93.

20. Avery K, Donovan J, Peters TJ, Shaw C, Gotoh M, Abrams P. ICIQ: a brief and robust measure for evaluating the symptoms and impact of urinary incontinence. Neurourol Urodyn. 2004;23(4):322-30.

21. Bristol Urological Institute. ICIQ-lower urinary tract symptoms quality of life. Available at: http://www.iciq.net/ICIQ.LUTSqolmodule.html. Accessed 20 May 2016.

\section{Submit your next manuscript to BioMed Central and we will help you at every step:}

- We accept pre-submission inquiries

- Our selector tool helps you to find the most relevant journal

- We provide round the clock customer support

- Convenient online submission

- Thorough peer review

- Inclusion in PubMed and all major indexing services

- Maximum visibility for your research

Submit your manuscript at www.biomedcentral.com/submit 